\title{
ON $E$-GROUPS IN THE SENSE OF PENG
}

\section{by HERMANN HEINEKEN}

(Received 23 March, 1988)

The groups $G$ of the title are those in which $E_{G}(x)=\left\{y \mid y \in G,\left[y,{ }_{n} x\right]=1\right.$ for some $n$ \} is a subgroup for every $x$ in $G$. We show that the quotient group $G / F(G)$ is rather restricted for finite $E$-groups; in particular, soluble finite $E$-groups are of Fitting length 4 at most. Some criteria for infinite groups are given.

We write as usual $[x, y]=x^{-1} y^{-1} x y$ and define inductively $[x, 1 y]=[x, y]$ and $\left[x,_{n+1} y\right]=\left[\left[x,{ }_{n} y\right], y\right]$. T. A. Peng considered the sets $E_{G}(y)=\left\{x \mid x \in G,\left[x,{ }_{k} y\right]=1\right.$ for suitable $k\}$ and he called a group $G$ an $E$-group if and only if all of these sets $E_{G}(y)$ are subgroups. He was able to show, among other things, that extensions of nilpotent by abelian groups are $E$-groups and that certain nonabelian simple groups are $E$-groups. On the other hand, he gave an example of a group $G$ such that $G / F(G)$ is supersoluble and $G$ is not an $E$-group.

In this paper we want to continue, on the basis of Peng's results, the efforts towards a characterization of these groups. A main step in this is the proof that the class of finite $E$-groups is a formation (Theorem 1 ). Considering the way $G$ acts by conjugation on its chief factors, we find that chief factors of finite $E$-groups are abelian or simple and that finite soluble $E$-groups have Fitting length four at most (Lemma 7, Theorem 2). The last decades have led to a wealth of knowledge of finite simple groups. This allows us to decide which of them are $E$-groups.

After the completion of a first version of this article the author became aware of the fact that C. Casolo [2] had obtained partly the same results; Casolo was also able to show that finite groups are $E$-groups if and only if all their subnormalizers are subgroups. Therefore we have abbreviated parts of the manuscript to avoid duplication.

The author is grateful to T. A. Peng, Singapore, for the (unpublished) section of his $\mathrm{Ph}$.D. Thesis [9] concerned with simple $E$-groups. He is also indebted to C. Casolo for drawing his attention to several inaccuracies. Notation is mostly standard, with $F(G)$ the Fitting subgroup of $G$, and $N_{G}(U)$ the normalizer $\left(C_{G}(U)\right.$ the centralizer) of $U$ in $G$. If $A$ and $B$ are normal subgroups of $G$, we will also use $C_{G}(A / B):=\left\{x \mid x \in G, x^{-1} y x B=y B\right.$ for all $y$ in $A\}$; confusion does not seem probable by this modification.

1. Nilpotent normal subgroups and generalizations. We begin with a very general remark.

LEMma 1. Assume that $N$ is a normal subgroup of the group $G$ satisfying the following conditions:

(i) $N$ is an Engel group, that is $E_{N}(x)=N$ for all $x$ in $N$;

(ii) $G / N$ is an $E$-group;

(iii) if $x$ does not belong to $N$, the subgroup $K_{x}$ defined by $K_{x} / N=E_{(G / N)}(x N)$ is an E-group.

Then $G$ is an E-group.

Glasgow Math. J. 31 (1989) 231-242. 
Proof. We have to show that $E_{G}(x)$ is a subgroup for every $x$ in $G$. This follows from (i) if $x$ belongs to $N$ (with $E_{G}(x)=G$ ). If $x$ does not belong to $N$, we see from the canonical epimorphism of $G$ onto $G / N$ that $E_{G}(x)$ is contained in $K_{x}$. By (ii) and (iii), $E_{G}(x)=E_{K_{x}}(x)$ is a subgroup of $G$, and Lemma 1 is shown.

Our next result is a statement on one element $x$ and its set $E_{G}(x)$ but will prove useful in several cases.

LEMMA 2. If $N$ is a locally nilpotent normal subgroup of $G$ and $x$ is an element of $G$ such that $E_{(G / N)}(x N)=N_{(G / N)}(\langle x N\rangle)$, then $E_{G}(x)$ is a subgroup of $G$.

Proof. Choose first two elements $y, z$ from $E_{G}(x) \cap\langle x, N\rangle$ and consider $\langle x, y, z\rangle$. There are integers $r$ and $s$ such that $x^{r} y$ and $x^{s} z$ belong to $N$, and $\langle x, y, z\rangle=$ $\left\langle x, x^{r} y, x^{s} z\right\rangle=\left\langle x,\left\langle x^{r} y, x^{s} z\right\rangle^{\langle x\rangle}\right\rangle$. Since $\left\langle x^{r} y, x\right\rangle=\langle y, x\rangle$, we have that $x^{r} y$, and similarly $x^{s} z$, belong to $E_{G}(x)$, and $\left\langle x^{r} y, x^{s} z\right\rangle^{\langle x\rangle}$ is finitely generated and nilpotent. Now $\langle x, y, z\rangle$ is the extension of a nilpotent by an abelian group and therefore by Peng [8, Theorem 1, p. 321] an $E$-group. We have now that $E_{G}(x) \cap\langle x, y, z\rangle$ is a subgroup, and $y z^{-1}$ belongs to $E_{G}(x)$.

This shows $E_{G}(x) \cap\langle x, N\rangle$ is a subgroup, and $E_{G}(x) \cap\langle x, N\rangle=\left\langle x,\left(E_{G}(x) \cap N\right)\right\rangle$ is itself locally nilpotent; this follows, for instance, from $P$. Hall [4]. It must be therefore the (unique) locally nilpotent subgroup of $\langle x, N\rangle$ which is maximal with respect to containing $x$.

If $b$ is now any element of $E_{G}(x)$, we deduce from $\left[b,{ }_{n} x\right]=x^{-1}\left[b^{-1} x^{-1} b,{ }_{n-1} x\right] x$ that also $b^{-1} x^{-1} b$ belongs to $E_{G}(x)$. The canonical epimorphism of $G$ onto $G / N$ maps the set $E_{G}(x)$ into $E_{(G / N)}(x N)=N_{(G / N)}(x N)$, and we obtain

$$
\langle x, N\rangle=\left\langle b^{-1} x^{-1} b, N\right\rangle \quad \text { for } b \text { in } E_{G}(x) .
$$

We exploit the maximality of $E_{G}(x) \cap\langle x, N\rangle$ as maximal locally nilpotent subgroup of $\langle x, N\rangle$ and obtain

$$
\begin{aligned}
E_{G}(x) \cap\langle x, N\rangle & =E_{G}\left(b^{-1} x^{-1} b\right) \cap\langle x, N\rangle=E_{G}\left(b^{-1} x b\right) \cap\langle x, N\rangle \\
& =E_{G}\left(b^{-1} x b\right) \cap\left\langle b^{-1} x b, N\right\rangle=b^{-1}\left(E_{G}(x) \cap\langle x, N\rangle\right) b .
\end{aligned}
$$

We have shown

$$
E_{G}(x) \subseteq N_{G}\left(E_{G}(x) \cap\langle x, N\rangle\right) .
$$

The other inclusion is trivial, so we have $E_{G}(x)=N_{G}\left(E_{G}(x) \cap\langle x, N\rangle\right)$, and Lemma 2 is proved.

COROLLARY 1. If $N$ is a locally nilpotent normal subgroup of $G$ such that $G / N$ is a free group, then $G$ is an E-group.

This follows directly from Lemma 2.

CoRollary 2. (Messingschlager [6, Satz 1]). If $N$ is a locally nilpotent normal subgroup of $G$ and all subgroups of $G / N$ are soluble $T$-groups, then $G$ is an E-group. 
Proof. $G / N$ is metabelian by Robinson [10], so it is an $E$-group by Peng [7, Theorem 1, p. 321]. Locally nilpotent $T$-groups are dedekind groups, and so $\langle x N\rangle$ is subnormal in $E_{(G / N)}(x N)$ and, again by the $T$-group property, even normal; $E_{(G / N)}(x N)=$ $N_{(G / N)}(\langle x N\rangle)$. Now the Corollary follows from Lemma 2.

Remark. Corollary 2 is no longer true if only $G / N$ itself is assumed to be a soluble $T$-group; this is shown by Example 1.

2. Finite nilpotent normal subgroups. In this section statements are deduced about quotient groups. The first statement is on sets $E_{G}(x)$.

Lemma 3. Denote by $K$ a finite normal subgroup of the group $G$ which is contained in the nilpotent normal subgroup $N$ of $G$. Then, for all $x$ in $G$, we have $E_{(G / K)}(x K) \cap$ $(N / K)=\left(E_{G}(x) K \cap N\right) / K$.

Proof. It suffices, by an obvious induction argument on the order of $K$, to prove the Lemma for the case when $K$ is contained in $Z(N)$. So now $K$ is finite and abelian, and we have

$$
K=\left(K \cap E_{G}(x)\right) \times L,
$$

where $L=[x, L]$. We deduce

$$
\left(E_{G}(x) \cap N\right) K=\left(E_{G}(x) \cap N\right) \times L
$$

and both factors are normalized by $x$.

Assume now the existence of an element $v$ in $N$ such that $v K$ belongs to $E_{(G / K)}(x K)$ and $[v, x]$ belongs to $\left(E_{G}(x) \cap N\right) K$. We have a unique description

$$
[v, x]=a b \text { with } a \text { in } E_{G}(x) \cap N \text { and } b \text { in } L .
$$

There is an element $t$ in $L$ such that $b=[t, x]$ and we obtain $\left[v t^{-1}, x\right]=t[v, x] t^{-1}\left[t^{-1}, x\right]=$ $[v, x][t, x]^{-1}=a$, and so $v t^{-1}$ belongs to $E_{G}(x) \cap N$, and $v$ belongs to $\left(E_{G}(x) \cap N\right) K$. For a general element $w K$ of $E_{(G / K)}(x K)$ there is a number $s$ such that $\left[w,{ }_{s} x\right]$ belongs to $\left(E_{G}(x) \cap N\right) K$, and the previous argument shows (by induction on the minimum number s) that

$$
E_{(G / K)}(x K) \cap(N / K) \subseteq\left(E_{G}(x) \cap N\right) K / K=\left(E_{G}(x) K \cap N\right) / K .
$$

Since the other inclusion is obvious, we have the desired equality.

If $N$ is a normal subgroup of $G$ and $y$ belongs to the subgroup $E_{G}(x)$, then we obtain at once $y^{-1}\left(E_{G}(x) \cap N\right) y=E_{G}(x) \cap N$. The next Lemma shows that imposing this condition sometimes allows one to pass to quotient groups.

LEMma 4. If $N$ is a finite nilpotent normal subgroup of the group $G$ and $x$ is an element of $G$ such that

$$
E_{G}(x) \cap N=y^{-1}\left(E_{G}(x) \cap N\right) y \quad \text { for all } y \text { in } E_{G}(x),
$$

then $E_{(G / N)}(x N)=E_{G}(x) N / N$. 
Proof. Again one inclusion is trivial:

$$
E_{G}(x) N / N \subseteq E_{(G / N)}(x N) .
$$

For the other inclusion we first collect some facts. By Peng [7, Theorem 1, p. 321] $V_{0}=E_{G}(x) \cap N$ is a subgroup of $N$; it is by hypothesis normalized by all elements of the set $E_{G}(x)$. Consider a product $a b$ with $a$ in $E_{G}(x) \cap N$ and $b$ in $E_{G}(x)$. Then $[a b, x]=b^{-1}[a, x] b[b, x]$ is an element of the same form, and if $\left[b_{n}, x\right]=1$, we have $\left[a b,{ }_{n} x\right] \in E_{G}(x) \cap N$. So $a b$ belongs to $E_{G}(x)$; we have shown

$$
E_{G}(x)=E_{G}(x)\left(E_{G}(x) \cap N\right)=\left(E_{G}(x) \cap N\right) E_{G}(x) .
$$

Beginning with $V_{0}$, we define a sequence of subgroups of $N$ by

$$
V_{i+1}=N_{G}\left(V_{i}\right) \cap N \text {. }
$$

It is shown by induction on $i$ that all $V_{i}$ are normalized by all elements of $E_{G}(x)$. Conjugation by $x$ induces in every quotient $V_{i+1} / V_{i}$ an automorphism without fixed points.

To derive a contradiction, we assume the existence of an element $v N$ in $E_{(G / N)}(x N)$ which is not contained in $E_{G}(x) N$. Since $\left[v,{ }_{n} x\right] N=N$ for suitable $n$, there exists an element $w=\left[v,{ }_{k} x\right]$ such that $[w, x]$ belongs to $E_{G}(x) N$ but $w$ itself does not. We choose an element $g$ in $N$ such that

$$
[w g, x]=c d \text {, }
$$

where $d$ belongs to $E_{G}(x)$ and $c$ belongs to the minimal possible $V_{i}$. Let $i \neq 0$. Since conjugation by $x$ induces a fixed point free automorphism on $V_{i} / V_{i-1}$, there is an element $f$ such that

$$
(w g)^{-1}[f, x](w g) c \quad \text { belongs to } V_{i-1} \text {, }
$$

so

$$
[f w g, x]=(w g)^{-1}[f, x](w g)[w g, x]=(w g)^{-1}[f, x](w g) c d \in V_{i-1} E_{G}(x)=E_{G}(x) V_{i-1},
$$

contrary to the minimality of $i$. This shows that we can find an element $r$ in $N$ such that [wr, $x$ ] belongs to $E_{G}(x) V_{0}=E_{G}(x)$, and $w N$ is contained in $E_{G}(x) N$, contrary to our choice of $w$. This proves the missing inclusion

$$
E_{(G / N)}(x N) \subseteq E_{G}(x) N / N
$$

and proves the Lemma.

We are now able to state our first statement on quotients of $E$-groups.

Lemma 5. The following statements on the finite group $G$ and its nilpotent normal subgroup $N$ are equivalent:

(i) $G$ is an $E$-group,

(ii) (a) $E_{G}(x) \cap N=y^{-1}\left(E_{G}(x) \cap N\right) y$ for all $y$ in $E_{G}(x)$,

(b) $G / N$ is an $E$-group. 
Proof. If (i) is true, $E_{G}(x)$ is a subgroup and $y^{-1} E_{G}(x) y=E_{G}(x)$ for all $y$ in $E_{G}(x)$ and (ii)(a) follows. Since by Lemma 4 we have $E_{(G / N)}(x N)=E_{G}(x) N / N$, also (ii)(b) is true. So (ii) follows from (i).

Now let $G$ be a finite group satisfying (ii). Then (ii)(a) and Lemma 4 yield

$$
E_{(G / N)}(x N)=E_{G}(x) N / N,
$$

and by (ii)(b) we know that $E_{G}(x) N / N$, and so also $E_{G}(x) N$, are groups. Again by (ii)(a) we have

$$
E_{G}(x) \subseteq E_{G}(x) N \cap N_{G}\left(E_{G}(x) \cap N\right)=L,
$$

say, and $L$ possesses the normal subgroups $E_{G}(x) \cap N$ and $L \cap N$.

The above inclusion is equivalent to

$$
E_{G}(x)=E_{L}(x),
$$

and $x$ induces in $(L \cap N) /\left(E_{G}(x) \cap N\right)$ by conjugation an automorphism without fixed points, so every element of $(L \cap N) /\left(E_{G}(x) \cap N\right)$ is a commutator $\left[x\left(E_{G}(x) \cap N\right)\right.$, $\left.u\left(E_{G}(x) \cap N\right)\right]$ where $u$ is taken from $L \cap N$.

Now

$$
E_{(G / N)}(x N)=E_{G}(x) N / N=L N / N \cong L /(L \cap N) .
$$

Since $E_{(G / N)}(x N)$ is a subgroup and $x N$ is a left Engel element of it, we have

$$
x N \in F\left(E_{(G / N)}(x N)\right)
$$

(see for instance Baer [1, Satz $\left.L^{\prime}\right]$ ), and using the canonical isomorphism, we obtain

$$
x(L \cap N) \in F(L /(L \cap N)) .
$$

We define $K /(L \cap N)=F(L /(L \cap N))$ and consider a Carter subgroup

$$
W /\left(E_{G}(x) \cap N\right) \text { of } K /\left(E_{G}(x) \cap N\right) \text {. }
$$

Since $K /(L \cap N)$ is nilpotent, we have $W(L \cap N)=K$, so there is an element $y$ of $W$ such that $y(L \cap N)=x(L \cap N)$ and $x^{-1} y$ belongs to $L \cap N$. As mentioned before, there is an element $u$ of $L \cap N$ such that

$$
x^{-1} y\left(E_{G}(x) \cap N\right)=[x, u]\left(E_{G}(x) \cap N\right)
$$

and

$$
y\left(E_{G}(x) \cap N\right)=u^{-1} x u\left(E_{G}(x) \cap N\right) .
$$

Now $u W u^{-1}$ contains $x$.

By the Frattini argument applied to the Carter subgroup $u W u^{-1} /\left(E_{G}(x) \cap N\right)$ of the normal subgroup $K /\left(E_{G}(x) \cap N\right)$ of $L /\left(E_{G}(x) \cap N\right)$ we find that

$$
N_{\left(L /\left(E_{G}(x) \cap N\right)\right)}\left(u W u^{-1} /\left(E_{G}(x) \cap N\right) K /\left(E_{G}(x) \cap N\right)\right)=L /\left(E_{G}(x) \cap N\right)
$$

and so also

$$
N_{L}\left(u W u^{-1}\right) K=L
$$


Let $T=N_{L}\left(u W u^{-1}\right)$. By construction, $T \subseteq E_{G}(x)$ and $T \cap N=E_{G}(x) \cap N$, since $x$ does not generate a nilpotent subgroup with any element of $N \cap L$ which does not belong to $E_{G}(x) \cap N$. Consideration modulo $E_{G}(x) \cap L$ shows that there is at most one coset $v\left(E_{G}(x) \cap L\right)$ which is contained in $v(N \cap L)$ and in $E_{G}(x)$, for given $v$. We derive from $T K=L$,

$$
T=E_{G}(x),
$$

and so $E_{G}(x)$ is a subgroup. This shows that (i) follows from (ii), and Lemma 5 is true.

We are now ready for the main statement of this section.

THEOREM 1. If $G$ is a finite $E$-group and $N$ is some normal subgroup of $G$, then $G / N$ is an E-group.

Proof. We choose a minimal supplement $U$ of $N$ in $G$, so that $U N=G$ and $U \cap N$ is contained in the Frattini subgroup of $U$ and hence nilpotent. Since subgroups of $E$-groups are $E$-groups, we have at once that $U$ is an $E$-group. Since (ii)(b) of Lemma 5 follows from (i), we obtain

$$
U /(U \cap N) \text { is an } E \text {-group, }
$$

and we obtain the statement of our Theorem from $U /(U \cap N) \cong U N / N=G / N$.

Remark. Theorem 1 is also a corollary of Casolo [2, Theorem 1.11].

Now finite $E$-groups form a formation, since this class is also closed with respect to subgroups and direct products.

3. Finite soluble $E$-groups. We exploit Theorem 1 to determine how $G$ acts by conjugation on its chief factors. This leads to a result comparable with Peng [7, Theorem 5].

Lemma 6. If $A / B$ is a chief factor of the finite soluble E-group $G$, then the quotient group $G / C_{G}(A / B)$ is isomorphic to the subdirect product of two groups $R$ and $S$ where

(i) the commutator subgroup $R^{\prime}$ of $R$ is cyclic of odd order and all Sylow subgroups of $R$ are abelian and

(ii) $S$ is isomorphic to one of the following groups:

(a) the trivial group,

(b) $\operatorname{SL}(2,3)$,

(c) the multiplicative group of the irregular nearfield of order 49,

(d) a generalized quaternion group $Q_{2 t}, t \geq 3$.

Proof. By Theorem 1 we may assume without loss of generality that $B$ is trivial. Then $A$ is an elementary abelian $p$-group for some prime $p$. We define $T$ by putting

$$
F\left(G / C_{G}(A)\right)=T / C_{G}(A) .
$$

It is well known that $T / C_{G}(A)$ is of order prime to $p$ in this case; and $A$ is a direct product 
of some minimal $T$-invariant subgroups $S_{i}$ where these subgroups $S_{i}$ can be chosen conjugate in $G$. Now the subgroups $C_{T}\left(S_{i}\right)$ are also conjugate in $G$, and

$$
C_{T}\left(S_{i}\right)=C_{T}(A)=C_{G}(A) .
$$

Since the orders of $A$ and $T / C_{T}(A)$ are relatively prime, we have

$$
E_{T}(x) \cap A=C_{T}(x) \cap A \quad \text { for all } x \text { in } T .
$$

Assume for the moment that there is an element $w$ of $T$ which is contained in $C_{T}\left(S_{i}\right)$ but not in $C_{T}\left(S_{j}\right)$. There is some element $g$ in $G$ such that $g^{-1} S_{i} g=S_{j}$, and (by Lemma 4) $g$ can be taken from $E_{G}(w)$. Now $E_{T}(w) \cap A=g^{-1}\left(E_{T}(w) \cap A\right) g$ leads to a contradiction. So we must have $C_{T}\left(S_{i}\right)=C_{T}(A)$ for all $i$. In the same way we argue for normal subgroups $D$ of $T$ such that $D^{\prime} \subseteq C_{G}(A) \subseteq D$, and we find that $C_{D}\left(M_{i}\right)=C_{D}\left(M_{j}\right)=C_{G}(A)$ for all minimal $D$-invariant subgroups $M_{j}$ of $A$. Since $D / C_{G}(A)$ is abelian, we may apply Schur's Lemma and obtain

(+) All abelian normal subgroups of subgroups of $T / C_{G}(A)$ are cyclic.

The nilpotent groups with this property are known. We obtain

(++) $F\left(G / C_{G}(A)\right)=T / C_{G}(A)$ is a direct product $H \times K$ such that

(i) $H$ is cyclic of odd order,

(ii) $K$ is a cyclic 2-group or a generalized quaternion group.

If, on the other hand, $Y / C_{G}(A)$ is a Sylow subgroup of $G / C_{G}(A)$ for a prime different from $p$, we have that $A$ splits into minimal $Y$-invariant subgroups, and the intersection of their centralizers coincides with $C_{G}(A)$. If $Y / C_{G}(A)$ is a Sylow 2-subgroup, we can only deduce that all elements of order 2 belong to the centre of $Y / C_{G}(A)$; for all other primes $Y / C_{G}(A)$ is abelian. Keeping this fact in mind together with the fact that $G / T$ is isomorphic to a subgroup of the outer automorphism group of $T / C_{G}(A)$, we obtain the result given in Lemma 6.

THEOREM 2. If $G$ is a finite soluble E-group, $G$ is contained in $\mathfrak{N}^{4}$. More precisely, all Sylow subgroups of odd order of $G / F(G)$ are abelian, all involutions of Sylow 2-subgroups of $G / F(G)$ are contained in the centre, and

$$
F(G) \supseteq\left(G^{\prime \prime}\right)^{4}\left[\left(G^{\prime \prime}\right)^{2}, G\right] .
$$

Proof. The second statement follows since it is true by Lemma 6 for all $C_{G}(A / B)$ with chief factors $A / B$, in particular $G / C_{G}(A / B)$ belongs to $\mathfrak{R}^{3}$. The statement on the Sylow subgroups is inherited by subdirect products and follows again from Lemma 6 .

Corollary. A finite soluble E-group is of p-length 2 for all primes.

This follows from $G \in \mathfrak{R}^{4}$.

4. Finite $E$-groups. In this section groups are finite and not necessarily soluble.

We begin with two remarks.

Lemma 7. (a) If the finite E-group $G$ possesses a subgroup $U$ and an element $x$ such that $x^{-1} U x$ is contained in $C_{G}(U)$, then $U$ is nilpotent. 
(b) Chief factors of finite E-groups are simple or abelian.

Proof. (a) Assume false: there is an element $y$ of $U$ which does not belong to $F(U)$. By definition, $x^{-1} U x$ and $x$ belong to $E_{G}(y)$, and since $E_{G}(y)$ is a subgroup so also does the subgroup $x x^{-1} U x x^{-1}=U$, a contradiction to $y$ not being contained in $F(U)$.

(b) now follows since quotient groups of finite $E$-groups are $E$-groups by Theorem 1 and nonabelian minimal normal subgroups are direct products of conjugate simple groups. By (a), there is only one such factor, and minimal normal subgroups which are nonabelian have to be simple.

The finite simple $E$-groups have been found out independently by Casolo [2] and the author. To avoid duplications, the results are now cited from Casolo [2]. $\mathrm{Sz}\left(2^{2 n+1}\right)$.

THEOREM 3. If $G$ is a finite nonabelian simple E-group, it is one of $\operatorname{PSL}\left(2,2^{n}\right)$,

For a proof see Casolo [2, Theorem 2.1].

In the case of groups $G$ with trivial centre we know that $G$ and the group $\operatorname{Inn}(G)$ of inner automorphism are isomorphic. For noncentral extensions we consider first subgroups of $\operatorname{Aut}(G)$.

Lemma 8. Let $G$ be isomorphic to $P S L\left(2,2^{n}\right)$ or $S z\left(2^{n+1}\right)$. A subgroup $K$ such that $\operatorname{Inn}(G) \subseteq K \subseteq \operatorname{Aut}(G)$ is an $E$-group if and only if $|\operatorname{Inn}(G)|$ and $|K: \operatorname{Inn}(G)|$ are relatively prime, except for $\operatorname{PSL}(2,8)$ and $\mathrm{Sz}(32)$, where $K=\operatorname{Aut}(G)$ may occur.

For a proof see Casolo [2, Proposition 2.5].

The particular form of the groups in Theorem 3 allows a statement about finite $E$-groups in general.

THEOREM 4. If $T$ is the maximal nilpotent normal odd order subgroup of the E-group $G$, then $G / T C_{G}(T)$ is a subdirect product of a soluble group with the direct product of some groups isomorphic to $\operatorname{SL}(2,5)$.

Remark. For the soluble factor of $G / T C_{G}(T)$ consult Lemma 6 and Theorem 2.

Proof. Assume first that we have a finite $E$-group $G$ such that $G / F(G)$ is soluble and does not possess normal subgroups of odd order. Close inspection of Lemma 6 shows that $G / F(G)$ is a $(2,3)$-group where the Sylow 3-subgroup is abelian of exponent 3; the subgroup generated by all elements of order 3 is an extension of a nilpotent 2-group of class 2 by a 3-group, and the centre of the 2-group centralizes all elements of order 3 as well.

Now we consider finite $E$-groups $G$ with simple quotient $G / K$ and $K \supseteq T C_{G}(T)$ and the normalizer $M / F(G)$ of some Sylow 2-subgroup $S / F(G)$ of $G / F(G)$. The normalizer of $K S$ in $G$ is, in all cases except $G / K \cong P S L(2,4)$, a noncentral extension of $K S$ by a cyclic group of order $q-1>3$. By the preceding remark we are left with $G / K \cong$ $\operatorname{PSL}(2,4)$. 
Here Lemma 6(ii) calls for a central extension, and we find another normal subgroup $L$ between $K$ and $T C_{G}(T)$ such that $G / L \cong S L(2,5)$. Since $P G L(2,5)=$ $\operatorname{Aut}(P S L(2,4)) \cong S_{5}$ is not an $E$-group, a subdirectly irreducible nonsoluble quotient of $G / T C_{G}(T)$ must be perfect. The proof of Theorem 4 is complete.

For the prime 2 we have a corresponding statement.

THEOREM 5. If $S$ is the maximal normal 2-subgroup of the finite E-group $G$, then $G / S C_{G}(S)$ is a subdirect product of (i) a metabelian group, and (ii) groups isomorphic to some PSL $\left(2,2^{n}\right)$ or $\mathrm{Sz}\left(2^{2 n+1}\right)$,

Proof. Theorem 5 follows directly from Theorem 1 and Theorem 2 for part (i), keeping in mind that $G / S C_{G}(S)$ has only trivial normal 2-subgroups. Part (ii) follows from Lemma 8 together with cyclicity of all Sylow subgroups of odd order (which is not true for $\operatorname{Aut}(\operatorname{PSL}(2,8))$ and $\operatorname{Aut}(\mathrm{Sz}(32))$ leading to a contradiction to Lemma 6(i)).

Taking into account what we found at the beginning, we obtain with Lemma 8

Proposition 1. If $G$ is a locally finite group with normal 2-subgroup $N$ such that $G / N$ is either

(i) one of the groups $\operatorname{PSL}\left(2,2^{n}\right), \mathrm{Sz}\left(2^{2 m+1}\right)$

or

(ii) the direct product of two groups $\operatorname{PSL}\left(2,2^{n}\right), \mathrm{Sz}\left(2^{2 m+1}\right)$

such that the common prime divisor of the orders of both of them is only 2 , then $G$ is an E-group.

Proof. It suffices to assume that $G$ is finite since the property of being an $E$-group is decided by the three-generator subgroups.

We have to show, for elements $x$ outside $N$, that $E_{G}(x)$ is a subgroup. For elements of odd order this follows from $E_{G / N}(x N)=N_{G / N}(x N)$ with Lemma 2; for elements of order a power of 2 we have that $E_{G / N}(x N)$ is normalizer of a Sylow 2-subgroup either of $G / N$ or of one of its two direct factors, and we apply Lemma 1 . For elements $x N$ of $G / N$ which are of even order but not of order a power of 2, we have again that $E_{G / N}(x N)$ is a subgroup and $E_{\langle x, N\rangle}(x)=E_{\langle x, N\rangle}\left(x^{2 i}\right)$ is a subgroup for every $i$, and finally $E_{G}(x)$ is a subgroup.

The following is an indication for the situation in the general case.

Proposition 2. Assume that the finite group $G$ and its subgroups $N$ and $U$ satisfy the following conditions:

(i) $N$ is a nilpotent normal subgroup of $G$ and $G / N=S L(2,5)$,

(ii) $U N=G$ and $U \cap N=1$,

(iii) $\bigcap_{y \in U}\left(C_{G}(y) \cap N\right)=C_{G}(x) \cap N$, where $x$ is the central element of $U$ which is different from the identity.

Then $G$ is an E-group.

Proof. We state first that $x$ is well defined since $U \cong U N / N \cong S L(2,5)$. Condition (iii) implies that $C_{G}(y) \cap N \supseteq C_{G}(x) \cap N$ for all $y$ in $U$. If $z$ is an element of order 4 in $U$, 
then $z^{2}=x$ and so

$$
C_{G}(z) \cap N=C_{G}(x) \cap N .
$$

We can apply now Lemma 4 and we obtain that $E_{G}(z) \cap N$ is a subgroup; in particular, $E_{G}(z)=E_{U}(z) \times\left(C_{G}(z) \cap N\right)$.

In case of an element of odd order $t$ we use Lemma 2.

5. Examples. Our first example is connected with Corollary 2.

EXAmple 1. The smallest field $F$ of characteristic 5 possessing a divisible 2-group $M$ as subgroup of the multiplicative group can be described as the set theoretical union of fields $G F\left(5^{2^{k}}\right)$. The group

$$
D=\left\langle\left(\begin{array}{ccc}
a^{-1} & 0 & 0 \\
0 & a & 0 \\
0 & 0 & 1
\end{array}\right) \text { for all } a \in M,\left(\begin{array}{ccc}
0 & 1 & 0 \\
1 & 0 & 0 \\
0 & 0 & 1
\end{array}\right)\right\rangle
$$

is a $T$-group but

$$
W=\left\langle\left(\begin{array}{ccc}
a^{-1} & 0 & b \\
0 & a & c \\
0 & 0 & 1
\end{array}\right) \text { for all } a \in M, b, c \in F,\left(\begin{array}{ccc}
0 & 1 & 0 \\
1 & 0 & 0 \\
0 & 0 & 1
\end{array}\right)\right\rangle
$$

is an extension of an elementary abelian group by a group isomorphic to $D$ and is not an $E$-group: the set

$$
E_{W}\left(\left(\begin{array}{rrr}
0 & 1 & 0 \\
1 & 0 & 0 \\
0 & 0 & 1
\end{array}\right)\right) \supseteq\left\{D,\left(\begin{array}{rrr}
1 & 0 & b \\
0 & 1 & +b \\
0 & 0 & 1
\end{array}\right) \text { for all } b \text { in } F,\left(\begin{array}{lll}
0 & 1 & 0 \\
1 & 0 & 0 \\
0 & 0 & 1
\end{array}\right)\right\}
$$

is not $W$ but generates $W:\left(\begin{array}{lll}1 & 1 & 1 \\ 0 & 1 & 0 \\ 0 & 0 & 1\end{array}\right)$ does not belong to the set. However, the slight
modification

$$
V=\left\langle\left(\begin{array}{ccc}
a^{-1} & 0 & b \\
0 & a & c \\
0 & 0 & 1
\end{array}\right) \text { for all } a \text { in } M \text { and } b, c \text { in } F,\left(\begin{array}{rrr}
0 & -1 & 0 \\
1 & 0 & 0 \\
0 & 0 & 1
\end{array}\right)\right\rangle
$$

is an $E$-group; it is a Frobenius group.

Finiteness in Theorem 1 is indispensable. The following is a "rather small" example to show this.

EXAmple 2. The following group is a (soluble) $E$-group with a quotient group which is not an $E$-group.

$$
R=\left\langle a, b, c, d \mid a^{2}=b^{2}=c^{3}=d^{3}=(a c)^{2}=1,[c, d]=[a, d]=b c b d^{-1}=1\right\rangle .
$$


The subgroups $\langle c, d\rangle$ and $\left\langle(a b)^{4}\right\rangle$ are normal subgroups of $R$, and $R /\left\langle(a b)^{4}\right\rangle$ is not an $E$-group, since the Fitting quotient is a dihedral group.

The Fitting subgroup of $R$ is $\left\langle(a b)^{4}, d, c\right\rangle$. We have to check all elements outside to find out whether $R$ is an $E$-group, and we obtain

$$
\begin{aligned}
E_{R}(a) & =\langle a, d\rangle=E_{R}\left(a d^{i}\right), \\
E_{R}(b) & =\langle b, c d\rangle=E_{R}\left(b(c d)^{i}\right), \\
E_{R}\left((a b)^{2}\right) & =\langle a, b\rangle=E_{R}\left((a b)^{2+4 n}\right),
\end{aligned}
$$

and the remaining elements are conjugates of those already mentioned. So $R$ is an $E$-group as stated.

The upper bound for the Fitting length in Theorem 2 is attained. This in shown by

Example 3. The following finite soluble $E$-group is of exact Fitting length 4:

$$
S=\left\langle\left(\begin{array}{lll}
1 & 0 & a \\
0 & 1 & b \\
0 & 0 & 1
\end{array}\right) \text { for all } a, b \text { in } G F(7),\left(\begin{array}{rrr}
0 & -1 & 0 \\
1 & 0 & 0 \\
0 & 0 & 1
\end{array}\right),\left(\begin{array}{rrr}
1 & 3 & 0 \\
-1 & -2 & 0 \\
0 & 0 & 1
\end{array}\right)\right\rangle
$$

The construction is based on the nearfield of order $7^{2}$ (see Zassenhaus [11, p. 217]).

Remark. Denote by $K$ the quotient group $F / F^{p} F_{3}$, where $F$ is the free group freely generated by four elements. $K$ admits a group of automorphisms isomorphic to $S / F(S)$ with $S$ from Example 3. Forming the corresponding split extension $T$ of $K$, we find that $T / \Phi(T)$ is an $E$-group but $T$ is not, since $T$ possesses an abelian normal subgroup $L$ such that $T / C_{T}(L) \cong S / S^{\prime \prime} \cong A_{4}$. So the class of finite $E$-groups is a non-saturated formation.

Example 4. In $D=S \times T$ where $S \cong T \cong P S L(2,4)$ we choose subgroups $U \subset S$, $V \subset T$ such that $U \cong V \cong S_{3}$, and elements $a, b$ of order 3 in $U, V$. Obviously $D$ is an $E$-group, and $\left(a^{-1}, b\right)$ is conjugate to $(a, b)$ in $E_{D}((a, b))=U \times V$.

In the wreath product $W=C_{2}$ wr $D$ we identify $D$ in the obvious manner with one complement of the base group. It is checked easily that $E_{W}((a, b)) \neq E_{W}\left(\left(a^{-1}, b\right)\right)$, so $W$ is not an $E$-group. This shows that the condition on the orders of the direct factors in Proposition 1 is indispensable.

\section{REFERENCES}

1. R. Baer, Engelsche Elemente Noetherscher Gruppen, Math. Ann. 133 (1957), 256-270.

2. C. Casolo, Finite groups in which subnormalizers are subgroups, to appear.

3. W. Gaschütz, Gruppen, in denen das Normalteilersein transitiv ist, J. Reine Angew. Math. 198 (1957), 87-92. $787-801$.

4. P. Hall, Some sufficient conditions for group to be nilpotent, Illinois J. Math. 2 (1958),

5. B. Huppert, Endliche Gruppen I (Springer, 1967). 
6. W. Messingschlager, E-Gruppen im Sinne von Peng, Diplomarbeit (Würzburg, 1987).

7. T. A. Peng, Finite soluble groups with an Engel condition, J. Algebra 11 (1969), 319-330.

8. T. A. Peng, On groups with nilpotent derived groups, Arch. Math. Basel 20 (1969) 251-253.

9. T. A. Peng, Ph.D. Thesis (Queen Mary College, University of London, 1965).

10. D. J. S. Robinson, Groups in which normality is a transitive relation, Proc. Cambridge Philos. Soc. 60 (1964), 21-38.

11. H. Zassenhaus, Über endliche Fastkörper, Abh. Math. Sem. Univ. Hamburg 11 (1936), $187-220$.

UNIVERSITÄT WÜRZBURG

Fed. ReP. Germany 